\title{
Reaction-diffusion fronts under stochastic advection
}

\author{
A. C. Martí, ${ }^{1}$ F. Sagués, ${ }^{2}$ and J. M. Sancho ${ }^{1}$ \\ ${ }^{1}$ Departament d'Estructura i Constituents de la Matèria, Universitat de Barcelona, Avenida Diagonal 647, E-08028 Barcelona, Spain \\ ${ }^{2}$ Departament de Química Física, Universitat de Barcelona, Avenida Diagonal 647, E-08028 Barcelona, Spain
}

(Received 12 February 1997)

\begin{abstract}
We study front propagation in stirred media using a simplified modelization of the turbulent flow. Computer simulations reveal the existence of the two limiting propagation modes observed in recent experiments with liquid phase isothermal reactions. These two modes respectively correspond to a wrinkled although sharp propagating interface and to a broadened one. Specific laws relative to the enhancement of the front velocity in each regime are confirmed by our simulations. [S1063-651X(97)10608-0]
\end{abstract}

PACS number(s): 47.27.Sd, 05.40.+j, 03.40.Kf

\section{INTRODUCTION}

Front propagation problems have been receiving increasing attention due to their relevance for nonequilibrium studies in a wide variety of fields [1]. Most of these studies are concerned with the velocity selection problem in deterministic systems or homogeneous medium (reaction-diffusion equations) [2]. Very recently front propagation in modulated [3] or stochastic media has been discussed [4].

Self-sustained chemical fronts propagating in quiescent media advance steadily at a velocity $v_{0}$, which results purely from the interplay between the chemical time scale $\tau_{\text {chem }}$ and the species diffusivity coefficient $D: v_{0} \sim\left(D \tau_{\text {chem }}^{-1}\right)^{1 / 2}$. However, in stirred media, additional length and time scales come to play a fundamental role in such a dynamical process. The interface, either wrinkled or broadened by the turbulent flow, propagates at a velocity $v_{T}$, which is enhanced relative to $v_{0}$ to an extent that depends not only on the invested energy but also on the coupling between the intrinsic length and time scales and those externally introduced by the stirring mechanism [5].

Needless to say, the addressed problem is of singular importance as a way to inquire about fundamental aspects of turbulent flows, but mainly for its practical relevance to combustion processes [6]. However, abundant studies motivated either way have not been able yet to resolve the existing considerable discrepancies between theoretical predictions and experimental results. These discrepancies concern fundamental issues such a turbulent propagation rates, velocity quenching effects, the role of turbulent spectra, etc. [5].

From the experimental point of view much has been gained recently by enlarging the scope of the problem when going from combustion processes to liquid phase reactions [7]. In this way experimentalists more confidently approach the set of simplifying assumptions invoked by most of the theoretical models. These later ones, based on either the socalled $G$ equation [8], passage-rate analysis [9], or scalecovariance laws [10] tend to focus on a single mode of turbulent propagation, i.e., that identified later on as based on the so-called mechanism. On the other hand, when resorting to computing simulations [11], numerical accuracy commonly restricts the examined scenario to small stirring intensities. On the whole, it is then likely to expect a future demand for more versatile computer approaches to compare with more general and better controlled experimental observations.

Advancing towards that direction, we report here on our computer results for interfaces propagating in stirred media. Stirring is mimicked by using a stochastic differential equation (SDE) based algorithm to simulate random, stationary, isotropic, and statistically homogeneous flows with wellprescribed properties [12]. In spite of this ad hoc procedure, the pair of experimentally identified limiting modes of front propagation in liquids phases [7], i.e., the aforementioned Huygens propagation (HP) and the distributed reaction zone (DRZ) regimes, are clearly exhibited in our simulations. In addition we do not suffer from strict limitations concerning stirring intensity, which enables us to look for broad range scalings of the turbulent propagation rates. Finally, the main advantage of our model is the easy control we gain over the flow parameters more relevant for front propagation in stirred media, i.e., the intensity $u_{0}^{2}$, spectra $E(k)$, spatial correlation length $l_{0}$, and time correlation $t_{0}$. In particular, in what follows we adopt the Kraichnan spectrum [13], describing a widely distributed band of excitations around a peak centered at some well-defined wave number $k_{0}$. At this point is worth emphasizing that the proposed methodology is totally adapted to a statistical description of turbulence [14] and belongs to the class of the so-called synthetic turbulence generating models [15].

The organization of this paper is as follows. The reactiondiffusion-advection model and the existing analytical prediction on front propagation in turbulent media are briefly reviewed in Sec. II. Our numerical results are presented and commented on according to the above theoretical predictions in Sec. III.

\section{TURBULENT FRONT MODEL AND ANALYTICAL PREDICTIONS}

A detailed presentation of the algorithm can be found in [12], but it is worth summarizing here its main ingredients:

(i) A stream function $\eta(\mathbf{r}, t)$ is introduced through a SDE written in terms of a zero mean Gaussian white noise $\boldsymbol{\zeta}(\mathbf{r}, t)$ :

$$
\frac{\partial \eta(\mathbf{r}, t)}{\partial t}=\nu \nabla^{2} \eta(\mathbf{r}, t)+Q\left[\lambda^{2} \nabla^{2}\right] \nabla \cdot \zeta(\mathbf{r}, t),
$$




$$
\left\langle\zeta^{i}\left(\mathbf{r}_{1}, t_{1}\right) \zeta^{j}\left(\mathbf{r}_{2}, t_{2}\right)\right\rangle=2 \epsilon_{0} \nu \delta\left(t_{1}-t_{2}\right) \delta\left(\mathbf{r}_{1}-\mathbf{r}_{2}\right) \delta^{i j}
$$

(ii) From the stochastic field $\eta$, the 2-d incompressible advecting flow is obtained as usual

$$
\mathbf{v}(\mathbf{r}, t)=\left(-\frac{\partial \eta(\mathbf{r}, t)}{\partial y}, \frac{\partial \eta(\mathbf{r}, t)}{\partial x}\right) .
$$

The two-dimensional space is chosen here for several reasons. First of all, it is chosen for the sake of simplicity of numerical simulations and secondly, because the essential trends of the phenomenology we want to study are independent of the spatial dimension. Recent experiments in chemical reactions in quasi-two-dimensional geometries [7] and theoretical analysis [10] support this assumption.

(iii) The whole procedure is Fourier transformed and accordingly discretized.

(iv) Within this scheme, the homogeneous, isotropic, and stationary velocity correlation $R(r, s)$ and the energy spectrum $E(k)$ can be properly defined and easily evaluated [12]. From these quantities, the stirring intensity $u_{0}^{2}$, and the integral time $t_{0}$ and length $l_{0}$ scales of the flow can be obtained using the following standard prescription:

$$
\begin{gathered}
u_{0}^{2}=R(0,0)=\int_{0}^{\infty} d k E(k), \\
t_{0}=\frac{1}{u_{0}^{2}} \int_{0}^{\infty} d s R(0, s), \\
l_{0}=\frac{1}{u_{0}^{2}} \int_{0}^{\infty} d r R(r, 0) .
\end{gathered}
$$
[13]:

The adopted Kraichnan spectrum has the following form

$$
E(k) \propto k^{3} \exp \left[-\lambda^{2} k^{2}\right] .
$$

This energy distribution is reproduced by prescribing

$$
Q\left(\lambda^{2} \nabla^{2}\right)=\exp \left(\frac{\lambda^{2} \nabla^{2}}{2}\right) .
$$

On the other hand, the basic statistical parameters of the flow introduced above expressed in terms of the input parameters, $\epsilon_{0}, \lambda, \nu$, read

$$
u_{0}^{2}=\frac{\epsilon_{0}}{8 \pi \lambda^{4}}, \quad t_{0}=\frac{\lambda^{2}}{\nu}, \quad l_{0}=\frac{\lambda \sqrt{\pi}}{2} .
$$

The next step is the formulation of the reaction-diffusionadvection scheme. We model this situation by means of a dynamical equation for the passive scalar field $\psi(\mathbf{r}, t)$ in a two-dimensional space:

$$
\frac{\partial \psi}{\partial t}=D \nabla^{2} \psi+f(\psi)-\nabla \cdot(\mathbf{v} \psi)
$$

where $f(\psi)$ is a nonlinear reaction term with at least two steady states. In our simulation we have taken $f(\psi)=\psi^{2}-\psi^{3}$. Our results are independent of the particular form of $f(\psi)$ chosen in the simulations.

In the absence of stirring $(\mathbf{v}=0)$, a stable planar front propagates the stable state $\psi=1$ ("products") into the invaded unstable one $\psi=0$ ("reactants"). The dimensionless front velocity and front thickness respectively read

$$
v_{0}=\sqrt{\frac{D}{2}}, \quad \delta_{0}=\sqrt{8 D} .
$$

Superposing stirring, two limiting regimes of front propagation are found in our simulations. First when the typical length scale of the flow $l_{0}$ is larger than the intrinsic one associated to the reaction-diffusion dynamics $\delta_{0}$, we observe a distorted front that propagates as a still rather sharp interface. Such a propagation mechanism is known in the combustion literature as the "thin flame," "flamelet," or "reaction sheet" regime [7]. Contrarily when $l_{0}$ is smaller than $\delta_{0}$, we observe what is referred to in the literature as a "DRZ" regime [7] i.e., a broadened front disrupted by the stirring flow. In both cases, turbulent propagation rates are larger than in quiescent media (parameter values of the presented simulations are quoted in the figure captions).

Each one of the previously identified conditions correspond to a specific propagation mechanism. The common rationale behind the "thin flame" mode is based on a HPlike argument: the front has the same local structure as in the planar case with normal velocity given by $v_{0}$, but its length increases due to wrinkling. This results on faster propagation velocities, in such a way that the relative increment in the velocity is equal to the relative increment of the length. On the other hand stirring is assumed to affect the velocity in the DRZ regime by solely increasing diffusive transport inside the broadened front. Note in passing that in neither case is any effect of the stirring on the intrinsic chemical time scale considered.

Before translating these arguments into quantitative terms let us define precisely the quantities involved. $L_{0}$ will be the length of the planar front, which coincides with the lateral length of the system. $L_{T}$ is the length of the curved front evaluated numerically as the length of the curve level of the field at the value $\psi=1 / 2 . D_{T}$ is the effective diffusion when turbulent flow is present. It is numerically evaluated by simulating the diffusion of a passive scalar under the influence of the same flow free of any chemical front. The velocity of the front under the turbulent flow $v_{T}$ is evaluated numerically as the increment of the products per unit of time and normalized to $L_{0}$.

According to our previous arguments we can establish the following two analytical results:

(i) In the HP regime,

$$
\frac{L_{T}}{L_{0}}=\frac{v_{T}}{v_{0}} \equiv S
$$

(ii) Contrarily, in the DRZ mode, we simply adapt the first fundamental relation of Eq. (9) to obtain

$$
S=\frac{v_{T}}{v_{0}}=\left(\frac{D_{T}}{D}\right)^{1 / 2} .
$$




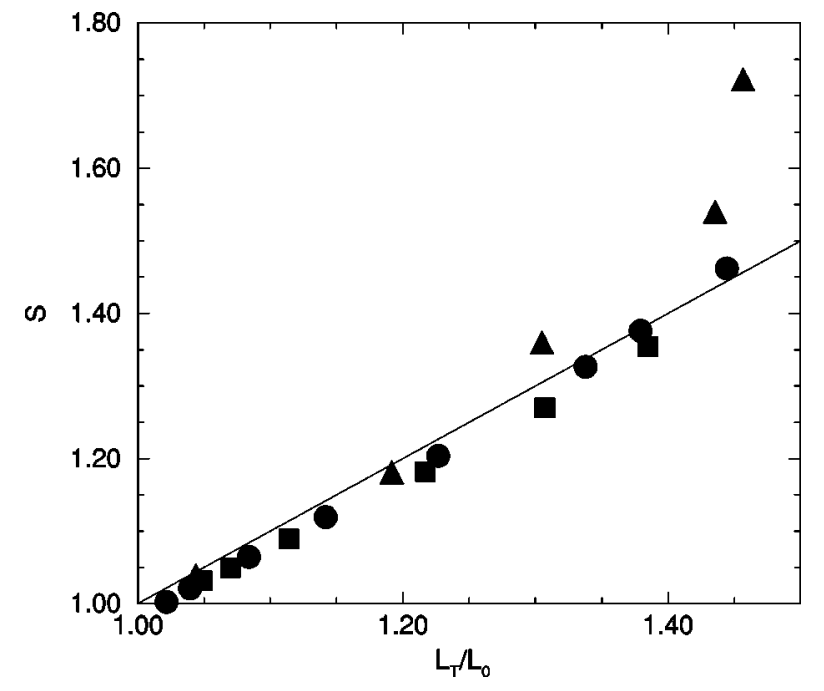

FIG. $1 . S$ vs $L_{T} / L_{0}$ [Eq. (10)] for turbulent flow (circles, $l_{0}=4.0, t_{0}=1.0$ ), random frozen (squares, $l_{0}=4.0$ ), and periodic eddies (triangles, $8 \times 8$ ). In all the simulations we have employed a square lattice of $128 \times 128$ points and unit spacing $\Delta r=0.5$ and $D=0.3$.

\section{NUMERICAL RESULTS AND COMMENTS}

The next and most involved step consists, however, in using Eqs. (10) and (11) to compare $v_{T}$, or its dimensionless form $S$, as a function of the stirring intensity $u_{0}^{2}$, or its dimensionless value $u_{0} / v_{0} \equiv Q$ with the results obtained from the numerical simulations of the Eq. (8). Let us consider separately the HP and DRZ regimes.

\section{A. HP mode}

In regard to the HP mode, our first task was to check relation (10). The collected data for the different values of $u_{0}^{2}$ are summarized in Fig. 1. For the sake of comparison, we include in this figure results obtained from two additional and somewhat related stirring conditions. The first one, hereafter referred to as frozen stirring, corresponds to a fixed configuration of the random flow. The second one, referred to in the following as periodic stirring, represents nothing but the limit of a deterministic and single scale flow, constructed from the single mode stream function

$$
\eta(x, y)=\eta_{0} \cos \left(\frac{n \pi x}{L}\right) \cos \left(\frac{n \pi y}{L}\right)
$$

representing a periodic array of $n \times n$ eddies, where $L$ is the system size. According to Fig. 1, the geometric argument leading to Eq. (10) seems well supported by our simulations with the simple exception of those situations involving very intense periodic flows, were the presence of overhangs (leading to the formation of isolated islands of reactants) is unavoidable. Note in this respect that the time evolution of those islands contributes positively to the computed velocity, measured as the time variation of the rate of occupation of the $\psi=1$ state, but negatively to the front length.

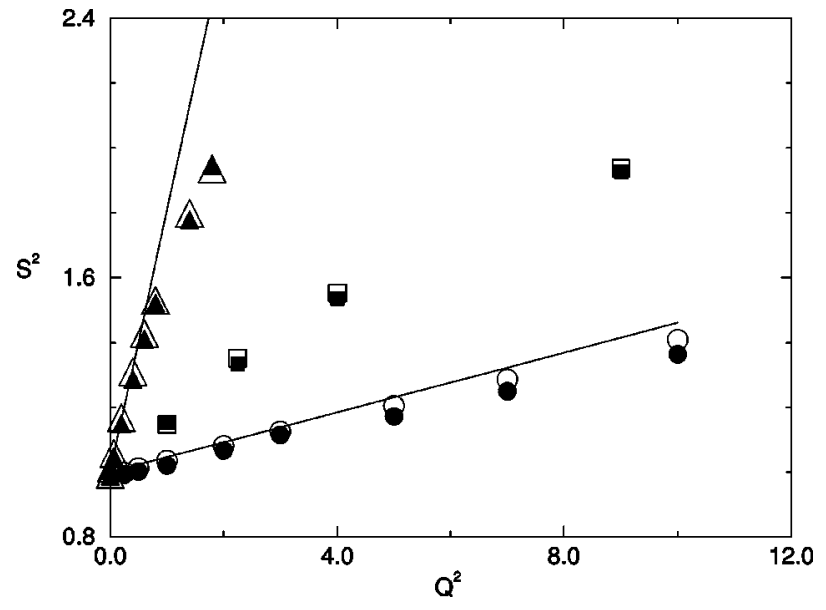

FIG. 2. $S^{2}$ vs $Q^{2}$ for the DRZ regime. Full symbols are simulation results of the front propagation while open symbols stand for the theoretical Eq. (11) using the effective turbulent diffusion (see text). Continuous lines correspond to the analytical predictions based on the perturbative results in Eqs. (13) and (14). Circles, squares, and triangles correspond to turbulent flow $\left(l_{0}=2.0\right.$, $\left.t_{0}=0.1\right)$, random frozen $\left(l_{0}=2.0\right)$ flows, and periodic eddies, respectively $(16 \times 16$ eddies $)(D=2.0)$.

\section{B. DRZ mode}

Results for the DRZ propagating mode are summarized in Fig. 2. Here again results for $S$ obtained by directly simulating the front propagation dynamics under the three different stirring mechanisms so far considered are plotted together. Computer results are compared with the corresponding theoretically predicted values based on Eq. (11) above. The agreement based on Eq. (11) is remarkable for the whole range of $Q$ values considered here and irrespective of the stirring flow. Explicit theoretical dependences of $D_{T}$ on $u_{0}^{2}$ valid respectively in two asymptotic cases are as follows.

(i) For a random flow in the weak stirring limit and small integral time $t_{0}$,

$$
D_{T}-D=u_{0}^{2} t_{0},
$$

a result obtained from Eq. (8) considering $\mathbf{v}$ as a Gaussian white noise [12].

(ii) For a periodic flow in the limit of small Peclet number [16],

$$
D_{T}-D \sim u_{0}^{2} / D .
$$

These two analytical results used in relation to Eq. (11) above lead to the theoretical estimates also shown in Fig. 2 (continuous lines).

A last remark concerning these two figures can clarify these results. The argument leading to Eq. (10) and Fig. 1 is purely kinematical or geometrical and only depends on the fact that the front is narrow enough. Thus different dynamical situations fit the prediction once this condition is fulfilled. 
Contrarily the argument for the DRZ (Fig. 2) case is dynamical so we observe different behaviors for the three different flows.

In summary, a recently proposed algorithm to model random stirring has been used in relation with front propagation. Although artificial in nature, such a model reproduces realistic scenarios of front propagation modes. These conclusions together with the versatile computer implementation of the proposed algorithm give us new hopes to address some of the open issues in the field of turbulent front propagation. Research in this direction basically aimed at finding the power law dependences of the propagating velocities on the stirring intensities and their fits to some of the most well- known relations proposed in the literature is presently being conducted and will be reported elsewhere.

\section{ACKNOWLEDGMENTS}

We acknowledge financial support by Comision Interministerial de Ciencia y Tecnologia, (Projects PB93-0769, PB93-0759) and Center de Supercomputació de Catalunya, Comissionat per Universitats i Recerca de la Generalitat de Catalunya. A.C.M. also acknowledges partial support from the CONICYT (Uruguay) and the Programa Mutis (ICI, Spain). We also acknowledge Professor P. Ronney for providing us with experimental data on turbulent chemical fronts.
[1] M. C. Cross and P. C. Hohenberg, Rev. Mod. Phys. 65, 851 (1993), and references therein.

[2] W. van Saarlos, Phys. Rev. A 39, 6367 (1989).

[3] J. Armero, A. M Lacasta, L. Ramirez-Piscina, J. Casademunt, J. M. Sancho, and F. Sagués, Europhys. Lett. 33, 429 (1996).

[4] J. Armero, J. M. Sancho, J. Casademunt, A. M. Lacasta, L. Ramirez-Piscina, and F. Sagués, Phys. Rev. Lett. 76, 3045 (1996).

[5] P.D. Ronney, in Modeling in Combustion Science, edited by J. Buckmaster and T. Takeno, Lectures Notes in Physics Vol. 449 (Springer-Verlag, Berlin, 1995).

[6] F.A. Williams, Combustion Theory, 2nd ed. (BenjaminCummins, Menlo Park, CA, 1985).

[7] P. D. Ronney, B. D. Haslam, and N. O. Rhys, Phys. Rev. Lett. 74, 3804 (1995).

[8] A. R. Kernstein, W. T. Ashurst, and F. A. Williams, Phys. Rev. A 37, 2728 (1988). For a recent review see also P. F.
Embid, A. J. Majda, and P. E. Souganidis, Phys. Fluids 7, 2052 (1995).

[9] A. R. Kernstein and W. T. Ashurst, Phys. Rev. Lett. 68, 934 (1992).

[10] A. Pocheau and D. Queiros-Condé, Phys. Rev. Lett. 76, 3352 (1996).

[11] D. C. Haworth and T. J. Poinsot, J. Fluid Mech. 244, 405 (1992).

[12] A. Careta, F. Sagués, and J. M. Sancho, Phys. Rev. E 48, 2279 (1993); A. C. Martí, J. M. Sancho, F. Sagués, and A. Careta, Phys. Fluids 9, 1078 (1997).

[13] R. H. Kraichnan, Phys. Fluids 13, 22 (1970).

[14] W. D. Mc Comb, Rep. Prog. Phys. 58, 1117 (1995).

[15] A. Juneja, D. P. Lathrop, K. R. Sreenivasan, and G. Stolovitzky, Phys. Rev. E 49, 5179 (1994); M. Holzer and E. D. Siggia, Phys. Fluids 6, 1820 (1994).

[16] H. K. Moffat, Rep. Prog. Phys. 46, 621 (1983). 